\title{
Medicina y literatura: los remedios literarios del Dr. Wilde
}

\author{
SANDRA GASPARINI Universidad de Buenos Aires, Argentina / sandra_gasparini@hotmail.com
}

\section{Resumen}

Eduardo Wilde, médico, escritor y político argentino nacido en Bolivia durante el exilio de sus padres, presidente en dos oportunidades (1880 y 1898) del Departamento Nacional de Higiene, se convirtió en sus textos en ese médico nuevo que debía luchar contra epidemias, lidiar a solas con la muerte y las tragedias íntimas de cada familia. Representándose como protagonista de esos relatos que combinan reflexión, anécdota, evaluación, descripciones impecables que rozan el naturalismo y el gótico en dos derivas diferentes crea un pivote perfecto para que esa masa textual que parece ir a la deriva de las digresiones o de los «relatos al caso» tenga un anclaje en la mirada médica que describe, analiza y diagnostica con cáustica ironía. El cruce de la experiencia médica sobre los cuerpos con lo autobiográfico es capitalizado por el narrador como un saber sobre lo natural y como una contienda contra la hipocresía del sentido común. El saber científico se incorpora como un discurso que explica el mundo hasta sus bordes aunque es limitado: la ciencia médica se presenta como sistema incompleto pero a la vez necesario para regular la muerte - la epidemia, la mortalidad infantil-. La lectura se centra en algunos textos de Tiempo perdido (1878) y Prometeo \& Cia (1899).

Palabras clave: medicina / literatura argentina / siglo XIX

\section{Medicine and Literature: the literary drugs of Dr. Wilde}

Abstract

Eduardo Wilde, Argentine physician, writer and politician born in Bolivia during his parents' exile, two-time president (1880 and 1898) of the National Department of Hygiene, became in his texts that new doctor who had to fight against epidemics and face death and the intimate tragedies of each family alone. By picturing himself as the protagonist of those stories that combine reflection, anecdote, evaluation as well as impeccable descriptions fairly close to naturalism and the gothic in two different drifts, he creates a perfect gadget to anchor digressions or «cases» to a medical eye that describes, analyzes and diagnoses with caustic irony. The narrator takes advantage of the intersection of medical experience on bodies and autobiographical memories as knowledge of the natural and as a fight against the hypocrisy of common sense. Scientific knowledge is incorporated as a discourse that explains the world to its limits although limited: medical science is presented as an incomplete system but at the same time necessary to regulate death - epidemics, child mortality-. The reading focuses on some texts from Tiempo perdido (1878) and Prometeo \& Cia (1899). 
El médico es un traductor, propone Mario Bunge en su «Introducción» a Filosofía para médicos (2012). «El médico intentará traducir los síntomas que siente el paciente a signos o indicadores objetivos (biomarcadores) de los procesos biológicos morbosos que le ocurren (materialismo)» (Bunge:15). El poeta es un fingidor, propone Fernando Pessoa: «Finge tan completamente/ que hasta finge que es dolor/ el dolor que en verdad siente/Y, en el dolor que han leído,/ a leer sus lectores vienen,/ no los dos que él ha tenido,/ sino sólo el que no tienen».' Un médico puede leer y traducir el dolor. Un poeta puede fingir dolor para ser leído; un dolor que es parte de su vida y que pasa a la literatura. En Eduardo Wilde se conjugaron ambas cosas: una vocación para transformar el dolor en materia narrable y la decisión de trasmutar la ciencia médica en observatorio de la sociedad.

En «Los dolores» (publicado originalmente en 1926) Viktor Von Weizsäcker sostenía que «el acto de curar solo consiste en un contacto entre dos personas y muchos de estos contactos existen en el juego, la pelea y el amor» (91). La primitiva acción curativa que implica imponer la mano en el sitio del dolor, en efecto, afirma, compromete una decisión: «al contemplar el dolor uno no puede permanecer inmóvil: o bien nos acercamos a él o nos apartamos. La inclinación hacia el dolor es en realidad el sentido de la elección de la profesión de médico (...) Convertirse en médico es entonces la inclinación ante el acto de dolor» (92). Claro que esta candorosa «inclinación» que, entre otras pulsiones de menor importancia, señala Von Weizsäcker como características de la práctica médica, trae a la mente, en el ámbito de la literatura argentina del entresiglo XIX/XX, la contrapartida de este deber ser: se trata, por citar un par de casos, de los médicos fáusticos y perversos de «El hombre artificial» (1910) de Horacio Quiroga o de los estudiantes de medicina como el protagonista de «Filarmonoterapia» (1879), de Elías F. Bori (seudónimo de Belisario F. Arana, 1856-1909), casi atraídos por el dolor de sus pacientes; médicos investigadores, sin embargo, en un rango diferente del que, narrativamente, le interesa a Wilde.

El Departamento Nacional de Higiene se creó en Argentina en 1880 y, tres años después, comenzó a funcionar la Asistencia Pública de Buenos Aires. Los ciclos epidémicos eran muy frecuentes en el espacio urbano y, frente a esto, se tomaron medidas de gobierno como la construcción de redes de agua potable y cloacas, además de la pavimentación de las calles que, junto con la exclusión y la vigilancia, redundaron en la reducción de la mortalidad. En los escritos de Eduardo Wilde, médico, escritor y político, presidente en dos oportunidades (1880 y 1898) del Departamento Nacional de Higiene, queda revelada esta fuerte preocupación. Se convirtió él mismo en sus textos en ese médico nuevo que debía luchar contra epidemias, lidiar a solas con la muerte y las tragedias íntimas de cada familia. Representándose como protagonista de esos relatos que combinan reflexión, anécdota, evaluación, descripciones impecables que rozan tanto el naturalismo como el gótico crea un pivote perfecto para que esa masa textual que parece ir a la deriva de las digresiones o de los «relatos al caso» tenga un anclaje en la mirada médica que describe, analiza y diagnostica con cáustica ironía.

La incorporación de la experiencia médica sobre los cuerpos a lo autobiográfico en Wilde es capitalizada como un saber sobre lo natural y como una contienda contra la hipocresía social. El saber científico ingresa como un discurso que explica el mundo hasta sus bordes aunque es limitado: la ciencia médica se presenta como sistema incompleto pero a la vez necesario para regular el avance triunfal de la muerte - la epidemia, la mortalidad infantil—. En esta lectura me centraré en algunos textos de Tiempo perdido (1878) y Prometeo \& Cia (1899), donde esa mirada médica emerge de manera más clara y se enmarca en una etapa de inauguración y otra de cierre. 


\section{Autor/doctor}

En Wilde la cuestión de la autoría aparece en primer plano. La pulsión autobiográfica, como lo ha sugerido Ricardo Rojas, es un eje de la escritura y ha jugado con ella desde la utilización de seudónimos en la prensa (como Baldomero Tapioca), que luego incluye en algunos relatos, hasta el uso político de un texto como Prometeo \& Cia, para realzar su figura de escritor cuando la de funcionario público había aparecido difamada. Si bien detrás de toda ficción se sitúan trazas de una vida y de una vida organizada en la relación causa efecto, el caso de Wilde agrega el plus de enfrentarse a un rompecabezas en el que lo vital emerge en la anécdota del viajero, del médico, prácticas que remiten a su figura pública y que se atomizan en fragmentos que, finalmente, van conformando una totalidad en el conjunto.

Pensar la categoría de autor moderno en Wilde implica tener en cuenta los géneros que transitó, su escritura que apenas roza lo ya huidizo, porque elige materias volátiles y cinéticas como la lluvia, el viaje, el agua. Y la de Wilde, a su vez, es una textualidad que se resiste a su análisis porque se presenta como inasible e itinerante.

Prometeo \& Cia (1899) coincide con un momento de descrédito político: había abandonado su cargo como Ministro del Interior de Juárez Celman en enero de 1889 y viaja con su mujer por Europa, Asia, Rusia y EE. UU., a partir de lo que surgen Viajes y observaciones: cartas a La Prensa, una serie de crónicas publicadas luego. En 1898 vuelve a la función pública durante la segunda presidencia de Roca como presidente del Departamento Nacional de Higiene y con la publicación de Prometeo pretende recuperar su figura de escritor a la vez que la de hombre público. Recuperar, digo, porque apenas graduado de médico en 1870 el periódico satírico El Mosquito celebra su popularidad y su participación en la epidemia de fiebre amarilla, que anunció en la prensa, cuestión que lo consolida definitivamente (Bruno 2011). Es posible intuir esta temprana voluntad intervencionista ya en el discurso pronunciado con motivo del premio recibido por su tesis. Allí ensaya un embate que continuará desde sus escritos literarios posteriores: critica a los médicos y les reprocha su falta de compromiso social con las instituciones (Bruno 2011:28). Y como hará otro médico y naturalista escritor, Eduardo L. Holmberg, les reclamará la difusión del saber y el apoyo oficial para sostener asociaciones vinculadas con la medicina. Es que la década de 1870 justamente es el terreno en el que se cocina el proceso modernizador que tomará impulso en los 8os y la disputa por los escasos espacios y la renovación que estos jóvenes médicos veían necesaria para ponerse a la altura de ese proceso generaron, además de discordia, las discusiones de 1874 en la Facultad de Medicina en las que participaron Eduardo L. Holmberg y José María Ramos Mejía. ${ }^{2}$

Cristina Iglesia (2010) ha planteado que la modernidad de Wilde reside en su capacidad para cuestionar los lugares comunes de la literatura de fines de siglo XIX y en su puesta a prueba de las posibilidades de la escritura como acto autónomo, es decir, su especificidad. Este mismo movimiento parece requerir un lector nuevo o renovado que sea cómplice o que responda a la interpelación de la ironía. ${ }^{3} \mathrm{Si}$ la fantasía científica en la década de 1870 formuló modelos posibles de sujetos competentes para construir la nación que se estaba proyectando hacia el decenio siguiente, el perfil de los nuevos actores que requiere la modernidad fue trazado de manera furtiva por estas ficciones, a través del uso de la ironía, el grotesco y la hipérbole. Desde el periodismo y la literatura, donde emergen y se transforman diversos géneros, se fantasea con el carácter modélico y social de esos personajes: el nuevo naturalista, el nuevo médico, el nuevo reporter, el nuevo viajero y el inventor (Gasparini). Esta postulación implicará reformulaciones que jugarán a interactuar con 
los sujetos reales representantes del antiguo orden que se quiere modificar. Wilde, justamente, se convierte él mismo en ese médico nuevo, en ese higienista que debe luchar contra epidemias o la inoperancia de sus colegas. Ese médico autor es un producto del permanente juego con la vida y la muerte que recrea en sus textos, desde los más tempranos, recopilados en Tiempo perdido (1878) hasta los tardíos de Prometeo \& Cia (1899).

\section{Lectura médica}

Es bueno que se sepa que no estoy inventando, pues el punto que toco en este momento pertenece esencialmente a la medicina. («Fisiología de la música», 1967:170)

Salessi y Nouzeilles han estudiado exhaustivamente la trama del higienismo en los textos del siglo XIX argentino. Salto ha analizado la matriz discursiva del «caso médico» en el marco del auge del higienismo en la Argentina de la década de 1880:

Novelas, informes y hasta manuales escolares compartían, en muchos casos, una trama narrativa común: el caso, un tipo de narración que, según un modelo originado en la casuística religiosa y desarrollado por la clínica, proveía una matriz para la representación ficcional de las tensiones entre grupos étnicos y culturales diversos. Hay, en la época, casos de «neurosis célebres», de «irresponsables», de personas y de personajes «sin rumbo» o que llevan las enfermedades «en la sangre». Casos que, a partir de identificar lo patológico, señalar lo curioso o puntualizar lo interesante de un individuo, construyen una historia de su vida como historia de una patología, historia que otorga un orden —una organización coherente con la visión del médico escritor- al desorden de los signos patológicos. (Salto 2004:115-116)

Si bien los «casos» que analiza Wilde en algunas crónicas y relatos no «enfatizaban la índole ejemplarizadora de ciertas patologías individuales — alcoholismo, irresponsabilidad, locura, hermafrodismo, etc.» (Salto:117) — siguen la matriz guiada por una mirada médica que observa, saca conclusiones y diagnostica. Esto ocurre al menos en dos textos recopilados en Tiempo perdido que juegan con esa posibilidad de manera explícita: «Fisiología de la Ristori» (1869) y «Fisiología de la música» (1872). En ese sentido, Wilde también se adelanta y a la vez se aparta de este cúmulo textual en el que abundarán también historias de vida e historias clínicas entramadas en ficciones.

Foucault buscó la genealogía de la mirada médica y encontró un giro significativo en el siglo XVIII francés, cuando surge la clínica, cerca de revolución de 1789. El XIX será el dominio de la anatomopatología y el XX, el de la anatomo-clínica. La clínica integra la mirada con el saber propio de los médicos. El método clínico postula que síntomas y signos tienen un significado transparente y la mirada médica se vuelve atenta y pretende descubrir las particularidades del individuo que manifiestan la enfermedad, ahora enunciable en su verdad. «La mirada clínica tiene esa paradójica propiedad de entender un lenguaje en el momento en que percibe un espectáculo» (Foucault:155): está vinculada a un silencio que le permite escuchar.

En «Fisiología de la Ristori» Wilde presenta a la célebre soprano italiana que había visitado Buenos Aires por ese entonces casi en el contexto de un caso médico. Lejos de escribir una reseña de espectáculo, elige auscultar, observar, evaluar y dar un diagnóstico. Así, describe los efectos de la interpretación trágica en el rostro de la cantante: se mete en su córnea, su pupila y todo lo 
que contribuya a "pintar» la cólera lírica. Es decir: busca los efectos del arte en el cuerpo, en la naturaleza (en todo está la naturaleza: es la reina absoluta, aun a despecho de la ciencia, como en «Tini»). Dolor y arte parecen unidos, y la transformación del dolor en literatura es lo que Wilde urde en su poética. A su vez, el arte de fingir dolor es el principio constructivo de Adelaida Ristori: sus brazos y cabeza echados hacia atrás, sus órbitas que se agrandan, los movimientos de su boca, la sangre que corre por sus arterias contribuyen a generar un efecto en los espectadores. Wilde ensaya una vivisección del hecho artístico: durante ese trance, solo en ese momento, el arte controla el cuerpo, que es su autómata. ${ }^{4}$ Es por eso que la Ristori solo puede ser observada, disfrutada como «cuadro vivo». Como en el método clínico, integra lo visible con lo enunciable.

El diagnóstico final se impone en la estructura del discurso médico: «La Ristori debe ser una mujer excesivamente nerviosa y debe haber gastado mucho talento de observación y mucho poder de imitación para haber llegado a copiar tan bien el efecto de la moral sobre el organismo humano» (Wilde 1967:51).

«Fisiología de la música» se ubica a mayor distancia de su objeto: un observador descreído y aparentemente pragmático (quien manifiesta que en tiempos juveniles ha decidido estudiar contaduría en lugar de música) lee en los acordes de piano ejecutados por Alfredo Napoleón posibles dislocaciones o fracturas de los dedos de sus manos. Es decir, practica un extrañamiento en el que la mirada médica pretende separar la melodía que lo conmueve de los movimientos de tendones y músculos que la producen. Es que la mirada médica, particular forma de la observación que tiene como fin convertir en objeto de estudio clínico a un sujeto según su etología, ensaya la restauración de un orden perdido, anterior al arte (dramático, musical), un orden natural que el arte aprovecha para provocar un efecto emocional en los espectadores. Así, estos dos estudios «fisiológicos» que toman su andadura de los ensayos científicos juegan a sorprender al lector con sus disparatados diagnósticos. Si, en líneas generales, la fisiología médica ${ }^{5}$ se ocupa de los conceptos y principios biofísicos y bioquímicos básicos para el funcionamiento de los aparatos y sistemas del cuerpo humano, los ensayos "fisiológicos» de Wilde apuntan a informar, divertir y divertirse a partir de la observación cuasi científica de fenómenos que no suelen ser objeto de la ciencia. Como el surrealismo traerá más adelante a la mesa de disección la máquina de coser y el paraguas, el doctor Wilde reúne materiales diversos para provocar la sorpresa de un lector más cercano al Monsieur Toutlemonde de Lucio V. Mansilla que al especializado.

Un pianista «que haga excesos de ejecución en el piano no es un músico, es un autómata sujeto a accesos febriles que hacen bailar sus nervios» (Wilde 1967:171), afirma el observador y describe la ejecución pianística según la fisiología médica (170-171). Wilde confiesa que su mirada médica surge de su incapacidad para producir música. Manifiesta conocer las reglas del juego, pero no saber jugarlo: y esa es, precisamente, la matriz de la mirada médica. El diagnóstico no siempre impide la enfermedad ni la muerte porque los procesos naturales que describe y estudia exceden el limitado conocimiento científico: definitivamente la ciencia positiva no lo explica todo. «Si tuviera yo el gusto de la música, cuánto placer no recogería que anda volando por el aire, en lugar de reducirme a la triste condición de hacer aritmética con las más dulces armonías» (172).

\section{El médico literato}

En «Los prosistas fragmentarios», capítulo de Los modernos publicado por primera vez en 1922, Ricardo Rojas plantea la importancia de lo que podríamos llamar el principio autobiográfico en 
la producción de Wilde: los textos que dejaron tanto él como los otros autores a los que llamara «prosistas fragmentarios» (Cané, L. V. Mansilla) son el pálido testimonio de esas vidas intensas. Como señala Fontana, para Rojas, decir «los prosistas fragmentarios» es lo mismo que decir «los novelistas frustrados» (29) y esa condición fallida se debe solo en parte a la «cultura ambiente», que presenta como embrionaria e improvisada y que en consecuencia genera proyectos de textos y no textos «completos». En lo que respecta a Wilde su figura de autor se construye alrededor de la de médico, en una retroalimentación constante en la que la ciencia le permite abordar la experiencia pero no agotar su sentido. La duda que el proceso modernizador instala en la escritura de Wilde se anuncia como una paradoja, la del desajuste que el saber científico y los avances tecnológicos van profundizando.

Si bien la deriva gótica o la fantástica no son el eje de sus narraciones, le sirven para plantear un desequilibrio entre deseo y deber, entre escritura y representación, entre masas y disponibilidad, entre autor y lector. Pezzoni observa que coexisten el Wilde humorista con el severo, que se queja y amonesta. Su declarativa búsqueda de «naturalidad» entra en colisión con su búsqueda retórica, como cuando afirma en «Tini» (su cuento más célebre) que no es posible decir el dolor y, a la vez, da unos rodeos muy recargados para enunciarlo.

La presencia de un narrador con un rol textual co-referencial respecto del rol social médico (Salto 2000) ocupa todo el espacio en «Tini». ${ }^{6}$ Este cuento recupera una de las representaciones de médico que más discuten algunas fantasías científicas de los 70s: la figura del «sujeto irradiante» (relación que Vezzetti ha calificado como jerárquica entre paciente receptor/médico «sujeto irradiante»). Opuesta a la figura materna, al principio, termina siendo deconstruida a lo largo del relato: no es posible encontrar una cura o llegar en el momento justo a salvar la vida del paciente. El médico, del lado de la naturaleza; la madre del niño, del lado de la religión. ${ }^{7}$ El relato de esa imposibilidad, desdoblado en un primer narrador en tercera persona - fagocitado al final por la primera, que remite fuertemente a la figura autoral—, se hace a través de una serie de consideraciones sobre el aprendizaje y la adquisición del lenguaje en los niños, la oposición entre la inocencia infantil y las convenciones sociales, esas que tanto repudia la prosa de Wilde. La naturaleza está puesta en primer plano en este relato, dado que es esta entidad abstracta y pánica la que diagnostica el crup (difteria) de Tini, en un verdadero ejercicio de prosa poética que toma como eje el nombre de la enfermedad, que parece onomatopeya del gorgorismo respiratorio: solo después de ese diagnóstico lírico llega el médico. A partir de aquí el relato se escande al ritmo de la respiración de Tini y su madre: ronquido, respiración anhelosa, suspiro continuado. El silencio equivale a la falta de respuestas de la ciencia médica ("su ciencia había medido el abismo» - Wilde 2005:97-). Al sujeto médico casi sacerdotal y profesional que realiza la traqueotomía sin mirar a los ojos al niño se oponen los practicantes que lo cuidan y caminan a su alrededor, temerosos de despertarlo. Finalmente el narrador se saca la máscara (o el barbijo) y se asume como médico narrador: «Cuando mi corazón se oprime al ver un niño rubio como tú ¿es tu mano pequeña la que me lo aprieta desde el otro mundo?» (99). El rodeo llevaba a tocar la cuerda sensible del lector al exhibir el enlace autobiográfico o al impostarlo: o bien Tini es la representación de todos los niños que Wilde no pudo salvar o es la ficcionalización de uno de los tantos episodios protagonizados por el célebre médico (en su autobiografía inconclusa en tercera persona se alude al carácter puramente ficcional del personaje y a las razones de su uso). ${ }^{8}$ El remate entre macabro y risueño del hallazgo fortuito del zapatito de Tini un año después de su muerte reaviva el uso de estrategias 
del fantástico que no se despliegan del todo sino en dosis homeopáticas: a partir de ese botín la madre «ve» el pie de Tini mostrando su dedo, materialización de la ausencia que la medicina no pudo evitar pero que la literatura repone con su letra.

Wilde no se detiene, sin embargo en el detalle escatológico o microscópico del naturalismo. La primera edición de «Tini» corresponde, cronológicamente, a lo que Laera ha periodizado como un primer momento de inflexión de esa estética en Buenos Aires, caracterizado por fuertes tomas de posiciones aun sin que se contara con novelas publicadas y que se extiende desde 1879 hasta 1881. Sin rumbo (1885), la tercera novela de Eugenio Cambaceres, con claras intervenciones en ese sentido, culmina con una escena donde la medicina también se muestra inerme frente a la misma enfermedad infantil, el crup. El dramatismo, que Wilde no había ahorrado, se intensifica en la pluma del novelista, quien - ya en otra etapa del naturalismo - no intentará conmover sino provocar rechazo frente a lo filial monstruoso, además de cargar tintas en la tragedia personal de Andrés, el protagonista, que desembocará en el suicidio —y el final de su efímera familia—. ${ }^{9}$ Si Andrea, su pequeña hija, parece un cordero degollado luego de la traqueotomía inútil practicada por el médico es porque tanto el discurso científico como el religioso no pueden aliviarle los estragos que el escepticismo - inducido por apropiaciones muy personales de las lecturas Schopenhauer- ha hecho en él. Wilde, con matices, señala los límites de la ciencia médica pero, a través de "Tini», aporta una excepción a la regla: en algunos casos los médicos son tan víctimas de las limitaciones de la farmacología y del triunfo de la naturaleza como los pacientes y sus familiares.

\section{El médico forense de la sociedad porteña}

El final melodramático de «Tini» se enlaza con otras estrategias que Wilde utiliza frecuentemente: la animización de los objetos y el recuerdo lateral que ilumina un texto. Voy a referirme a la primera y a su uso en «La primera noche en el cementerio».

Los cuerpos muertos y los objetos hablan en los textos de Wilde. La materialidad se impone: ¿cuánto deberá medir el féretro que reciba al cadáver? Tanto en «Recuerdo al caso» (donde la memoria de la escena en la que acompaña a una muchacha mientras vela a un muerto — cuya materialidad describe con ojo clínico — sirve para iluminar la lectura de una novela de Podestá), como en «La primera noche en el cementerio $»^{10}$ hay prolijas y metódicas descripciones de cadáveres que recuerdan los informes médico legales de las autopsias," aunque en clave paródica. En el segundo texto, ya no se trata de la impotencia de la medicina para salvar una vida sino que directamente se recurre a la desacralización absoluta de los médicos. En especial, de un tipo de médico cuya figura se combate y se opone a la del narrador y que se caracteriza por su soberbia y la inutilidad de la farmacología que prescribe (Wilde 2005:124-125).

Wilde ironiza sobre las costumbres, la hipocresía social, la opinión pública y los estereotipos. En este texto se introduce la fantasía de manera explícita y con un personaje bien perfilado en los límites del gótico que hace ingresar al texto en el grotesco: el zombi («Me imagino, por una fantasía, un muerto vivo» - 125-). Se combina la descripción médica, histológica con la naturalista pero en un tono de humor negro. Cerca de este «muerto reciente» (claro: es su "primera noche») está la joven novia en descomposición y el narrador imagina una escena de sexo entre ambos, iniciada por una «ráfaga loca de sensualismo cadavérico» en el joven. Y este zombi también hace una apuesta política porque lejos de mantenerse quieto en su ataúd, en una versión anterior publicada en El Sud-americano en 1888, forma parte de la revuelta de los muertos vivos que animan la noche 
del cementerio, una representación de colorido social, una especie de carnaval de putrefacción donde no faltan los huelguistas de los sindicatos ni los frailes misioneros.

El narrador discute muy irónicamente con un interlocutor imaginario (¿la representación de un posible lector?) sobre la transmigración de las almas, en el marco de las disputas entre materialismo y espiritualismo de fines del XIX. ${ }^{12}$ Pero el eje de la ironía es en esta versión la vocación opositora del muerto reciente, que se oponía hasta a los candidatos de su partido. Como en una parodia de los reclamos que en esas décadas habían empezado a embarullar las calles el muerto vivo toma el periódico que le sostiene la cabeza, se inspira y al grito invocador de «iciudadanos!» convoca la resurrección de los muertos, que comienzan a levantarse de las tumbas. La pueblada de la fosa común y los habitantes de las casas aristocráticas se mezclan, la modernidad (telégrafo, prensa, teléfono) se opone a la antiguiedad clásica o a otras épocas más cercanas pero lejanas a la modernización técnica. Republicanos, realistas, feministas, anarquistas, todos reclaman sus derechos en esa Babel que tanto se parece a la Buenos Aires del referente histórico. Se dividen y están a punto de chocar fuerzas cuando la luna les muestra sus aspectos grotescos y cada uno vuelve a su tumba. El recurso fantástico de la vacilación se usa plenamente: ¿quién sabe si todo esto ha sido un sueño? De todos modos, el desorden en el cementerio persiste a la luz del día. Tal vez la impronta higienista de Wilde en tanto médico y político que alerta sobre los cementerios como factor de contaminación esté aquí presente pero en clave lúdica. La mirada médica y la del ironista político, también.

Así como, según Salto, «en las últimas décadas del siglo diecinueve las ficciones habrían provisto una trama narrativa a temas, tópicos y saberes científicos de escasa legitimidad disciplinaria al mismo tiempo que ciertos textos científicos se habrían apropiado de las estrategias discursivas de la ficción» (2000:104), «La primera noche...» reutiliza las estrategias descriptivas de la medicina legal que ya pusiera en práctica Wilde en tres textos pioneros publicados en Tiempo perdido para verosimilizar esta ficción gótica y aplicarle un efecto humorístico. ${ }^{13}$

Wilde ha elegido en sus textos conmover mediante el dolor de un ser ficcional, registrando en cada detalle lo que él supone una «sociedad enferma» para que así, construida como relato, "pued(a) encararse como una totalidad comprensible y dominable» (Pezzoni:277). Como médico que puede leer y traducir el dolor (el de los pacientes y el de la sociedad, denunciada en su oportunismo e hipocresía), arroja a quien quiera leerla la materialidad de los cuerpos en descomposición a través de ficciones grotescas e informes de autopsias. Como poeta que puede fingir que el dolor que siente no es solo literario increpa a los lectores «mentecatos» a través de «Tini» $y$ hace uso del melodrama con el objeto explícito de conmover. La mirada médica es, en definitiva, la arquitecta narrativa de un saber insuficiente, el de la medicina, que permite observar, clasificar y diagnosticar pero no siempre ser el ganador en la batalla contra la muerte y las rémoras de una sociedad que se quiere cambiar.

\section{Notas}

1 «Autopsicografía», en Fernando Pessoa (1998). Antología poética. El poeta es un fingidor. Madrid: Espasa Calpe.

2 Aunque Wilde reemplazó a su maestro, Montes de Oca, sus capacidades para enseñar Anatomía fueron cuestionadas por estos estudiantes rebeldes y renunció. Sin embargo, luego se sucederán algunos reconocimientos (entre otros, en 1874 es nombrado miembro titular de la Academia de Medicina).

3 Pezzoni (272) liga la ironía de Wilde a un escepticismo de pose; según él, Wilde descree solo de lo que le conviene.

4 «La Ristori [practica] a voluntad fenómenos que no dependen de ella, sino de la vida vegetativa a la cual la voluntad no manda, y sus sollozos y su respiración entrecortada por el poder 
del arte, es igual, exacta, idéntica a la que la naturaleza ejecuta cuando es presa de esas emociones» (Wilde 1967:49).

5 Ganong, W. F. (2010). Fisiología Médica. 23a Edición. México D.F.: Mcgraw-HILL Interamericana Editores, S.A. de C.V. 6 Publicado originalmente en El Diario, 19 de septiembre de 1881 y luego recogido en Prometeo \& Cia.

7 Y allí se juega una teoría del papel de las mujeres en la educación (recordemos las intensas discusiones en el Congreso durante 1883 antes de la aprobación final de la ley 1420 y la postura de Wilde en contra de la inclusión de contenidos religiosos en ella).

8 En Aguas abajo leemos sobre «Tini»: «(Lo escribió para probar a los mentecatos que sabía sentir: ellos lo ignoraban). El cuento publicado fue decisivo: nadie pudo leerlo sin llorar; y lo peor del caso es que el mismo autor, al corregir sus páginas dejaba caer en ella gruesas lágrimas, el niño imaginario se había vuelto real en su conciencia» (Wilde 1914:35-36).

9 Cambaceres irá más lejos en En la sangre (1887), su novela más naturalista: el factor hereditario — presente en el ADN de los inmigrantes-- será el verdadero monstruo.

10 «La primera noche en el cementerio» se publica en 1888. Tiene cuatro versiones, las dos primeras en Sudamérica y El Sud-americano y, cuando sale el libro, también las modifica para la primera y segunda edición, en 1899.

11 Salto afirma que a mediados de la década de 1870 «la autopsia era una práctica relativamente reciente que enfrentaba la práctica clínica al mismo tiempo que avalaba las especulaciones frenológicas y aportaba materiales a los incipientes devaneos criminalísticos» (92). El informe de la autopsia practicada por Mallo y Wilde en su condición de «médicos de sanidad del puerto central», excede los requerimientos descriptivos del informe anunciado y redunda en detalles explicativos que sostienen una trama de intriga y suspenso en torno de la muerte del individuo, propone Salto.

12 Algunos autores que en el último cuarto del siglo XIX contribuyeron con relatos y novelas a esta polémica son Eduardo L. Holmberg, Eduarda Mansilla, Raúl Waleis (anagrama de Luis V. Varela) y Carlos Monsalve, entre otros (Gasparini).

13 Los informes son tres y están incluidos en Tiempo perdido (1878), un volumen en el que conviven con reseñas y ficciones, entre otros géneros. Dos están firmados por Pedro Mallo y Eduardo Wilde: «Informe medicolegal relativo a una autopsia»e «Informe medicolegal sobre el estado mental de un individuo». El tercero, «Informe medicolegal sobre el estado mental de una señora», está firmado por Eduardo Wilde y Pedro Rosendi.

\section{Bibliografía}

Bunge, Mario (2012). Filosofía para médicos. Buenos Aires: Gedisa.

Bruno, Paula (2010). «Segundones cómplices. Acerca de la lectura de David Viñas sobre los gentlemen escritores del Ochenta». Prismas, (14)2. Web.

(2011). «Eduardo Wilde». Pioneros culturales de la argentina. Biografías de una época. Buenos Aires: Siglo XXI, 19-64.

Fontana, Patricio (2014). «Vida, escritura y sacrificio. Ricardo Rojas y los "prosistas fragmentarios"». Saga. Revista de Letras, 1, 20-40. Web.

Foucault, Michel (1963). El nacimiento de la clínica. Una arqueología de la mirada médica. Buenos Aires: Siglo XXI, 2004.

Gasparini, Sandra (2012). Espectros de la ciencia. Fantasías científicas de la Argentina del siglo XIX. Buenos Aires: Santiago Arcos.

Iglesia, Cristina (2010). «Eduardo Wilde: la literatura como autopsia del sentimiento», en Alejandra Laera, directora. El brote de los géneros, vol. 3 de la Historia crítica de la literatura argentina, Noé Jitrik, director general. Buenos Aires: Emecé, 233-255.

Laera, Alejandra (2004). El tiempo vacío de la ficción. Las novelas argentinas de Eduardo Gutiérrez y Eugenio Cambaceres. Buenos Aires: Fondo de Cultura Económica.

Nouzeilles, Gabriela (2000). Ficciones somáticas. Naturalismo, nacionalismo y políticas médicas del cuerpo (Argentina 1880-1910). Rosario: Beatriz Viterbo. 
Pezzoni, Enrique (1986). «Eduardo Wilde: lo natural como distancia». El texto y sus voces. Buenos Aires: Eterna Cadencia, 2009.

Rojas, Ricardo (1922). «Los prosistas fragmentarios». Los Modernos II. Historia de la literatura argentina. Buenos Aires: Kraft, 1957.

Salessi, Jorge (1995). Médicos maleantes y maricas: higiene, criminología y homosexualidad en la construcción de la nación argentina (Buenos Aires, 1871-1914). Rosario: Beatriz Viterbo.

Salto, Graciela (2000). «Los avatares literarios de la medicina legal: los informes medicolegales de Eduardo Wilde». Sabery Tiempo. Revista de Historia de La Ciencia, 10, 91-110.

(2004). «De las escenas de colegio a las escenas de hospital: la trama higienista en narraciones, anécdotas y casos», en María Silvia Di Liscia y Graciela Nélida Salto, editoras. Higienismo, educación y discurso en la Argentina (1870-1940). Santa Rosa: Universidad Nacional de La Pampa, 113-135.

Vezzetti, Hugo (1983). La locura en la Argentina. Buenos Aires: Folio.

Von Weizsäcker, Viktor (1926). Escritos de antropología médica. Buenos Aires: Libros del Zorzal, 2009. Traducción y compilación de Dorrit Busch.

Wilde, Eduardo (1878). Tiempo perdido. Buenos Aires: Huemul/Librería del Colegio, 1967. (1899). Prometeo \& Cia. Buenos Aires: Colihue/Biblioteca Nacional, Colección Los Raros. Estudio preliminar de Guillermo Korn, 2005.

(1914). Aguas abajo. Buenos Aires: Peuser. 